\title{
Der Macht des Wortes ausgesetzt, oder: Die Entdeckung der Fiktionalität in der griechischen Literatur der archaischen und klassischen Zeit
}

\section{Bernhard Zimmermann}

Die nun [die Musen] lehrten einst Hesiod den schönen Gesang, als er Schafe hütete am Fuße des heiligen Helikon.

Mich nun sprachen zuerst die Göttinnen an, die olympischen Musen, die Töchter des aigisschüttelnden Zeus: ,Hirten, die ihr auf freiem Feld lebt, übles Gesindel, Bauch nur, wir wissen viel Lügenhaftes zu sagen, das Wahrem ähnlich ist, wir wissen aber auch, sooft wir wollen, Wahres zu verkünden.' So sprachen sie, die Töchter des großen Zeus, die redegewandten, und sie gaben mir einen Stab, einen Zweig sprossenden Lorbeers, ihn zu tragen, einen ansehnlichen; und sie hauchten mir sehenden Gesang ein, damit ich besänge, was sein wird und was zuvor war.

Und sie hießen mich, zu besingen das Geschlecht der Seligen, die immer sind, sie selbst aber am Anfang und Ende immer zu besingen.

Doch was rede ich da um Eiche und um Stein herum?

Nun denn, mit den Musen wollen wir anfangen, die mit ihrem Gesang

dem Vater Zeus den großen Sinn erfreuen im Olympos, und wenn sie künden, was ist und was sein wird und was vorher war, einstimmig; unermüdlich fließt süß ihre Stimme aus ihrem Mund; und es lacht der Palast des Vaters Zeus, des heftig donnernden, aufgrund der Stimme der Göttinnen, die sich zart ergießt; es hallen die Gipfel des schneebedeckten Olymp und der Palast der Unsterblichen; die aber, ihre unsterbliche Stimme erklingen lassend, preisen das ehrfurchtgebietende Geschlecht der Götter zuerst, die am Anfang Gaia und Ouranos, die Erde und der Himmel, zeugten, und die Götter, die von ihnen abstammen, die Geber des Seienden [der Güter]. ${ }^{1}$

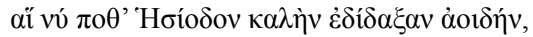

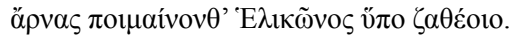

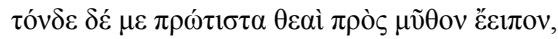

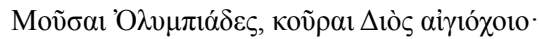
„,

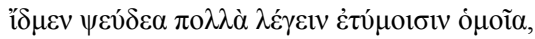

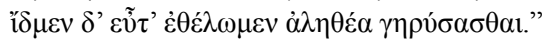

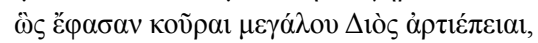

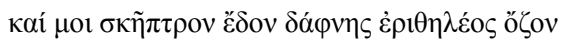

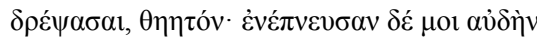

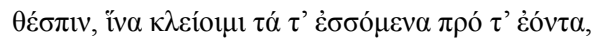

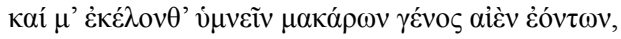

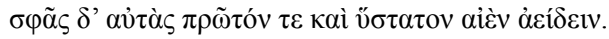

1 Alle Übersetzungen aus dem Griechischen in diesem Beitrag stammen vom Verfasser. 


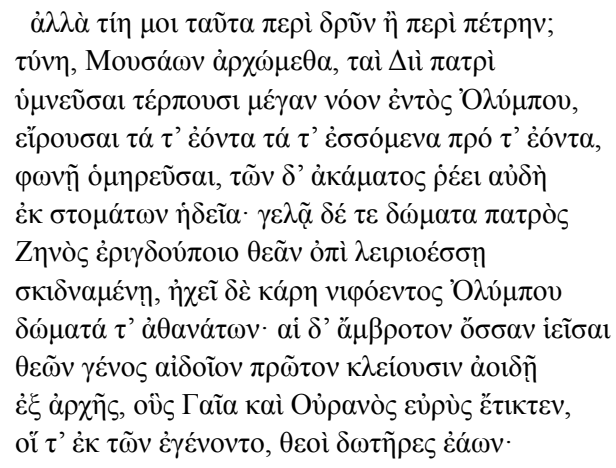

Die Verse 22-46 aus dem Proömium der Theogonie Hesiods (7. Jh. v. Chr.) berühren zentrale Themen der griechischen Dichtung der archaischen und klassischen Zeit, die die literaturtheoretische Diskussion - sei es implizit oder explizit - bis zur Poetik des Aristoteles umtrieb. Es tritt uns - im Gegensatz zum auktorialunpersönlichen Erzähler der homerischen Epen - eine Dichterpersönlichkeit entgegen, die sich selbst beim Namen nennt und in einer Dichterweihe von den Musen beauftragt wird, das zu besingen, was war und was sein wird. Nur der Dichter hat Zugang zu dem, was war, zu dem Schatzhaus der Erinnerung, zu den

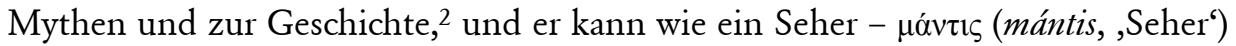
oder ,Musenherold' wird sich der Chorlyriker Pindar im 5. Jahrhundert nennen das singen, was erst kommen wird. Seine Gabe, die ihn von den anderen Menschen unterscheidet und ihn vor ihnen auszeichnet, erhält er von den Musen, also von den Göttinnen des Gesangs und der Dichtung. Doch gerade darin liegt eine Gefahr: Denn die Musen „wissen viel Lügenhaftes zu sagen, das Wahrem ähnlich ist“", sie wissen aber auch, wie sie sagen, sooft ihnen der Sinn danach

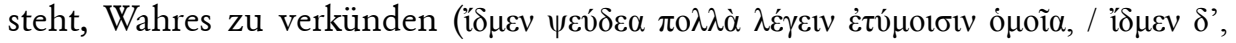

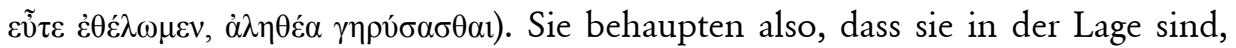
ihrem Medium, dem Dichter, Dinge einzugeben, die nicht wahr sind, ${ }^{3}$ und solche, die wahr sind - und dies ganz willkürlich, immer wenn sie es wollen. Die Heimtücke der Musen ist umso gravierender, als das Lügenhafte dem Wahren ähnlich ist, so dass es für den menschlichen Rezipienten keinen Hinweis darauf gibt, ob das, was er hört, falsch oder wahr ist.

Dieses rezeptionstheoretische Problem, die Unterscheidung von ,wahr ${ }^{6}$ und ,falsch', hängt damit zusammen, dass in der Dichtung der archaischen und klassischen Zeit bis ins 4. Jahrhundert v. Chr. Dichtung immer unter dem Gesichts-

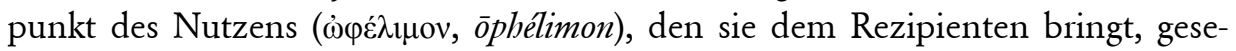
hen wurde, und dass man folglich - modern gesprochen - nicht zwischen Fachli-

2 Es muss betont werden, dass für griechische Autoren mindestens bis ins frühe 4. Jahrhundert v. Chr. kein Unterschied zwischen Mythos und Geschichte bestand.

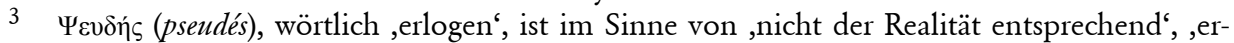
funden' zu verstehen. 
teratur und fiktionalen Texten unterschied (Rösler 1980). In der 405 v. Chr. aufgeführten, literaturkritischen Komödie Die Frösche des Aristophanes ist dieser didaktische Anspruch von Dichtung ein zentrales Anliegen. Der alte Tragiker Aischylos hält in dem Wettstreit um den Thron der tragischen Dichtung in der Unterwelt dem modernen, dekadenten Euripides vor, dass das Ziel guter Dichtung immer Erziehung und Unterweisung gewesen sei und auch künftig sein müsse (Verse 1030-1036):

Schau, wie von Anfang an die edlen Dichter nützlich waren.

Orpheus hat uns die Mysterien gezeigt und uns gelehrt, nicht zu töten,

Musaios Heilung von Krankheiten und Orakel, Hesiod

Ackerbau, Ernte der Früchte, das Pflügen. Und der göttliche Homer -

Woher hat der sein Ansehen und seinen Ruhm, wenn nicht davon, dass er

Nützliches lehrte: Schlachtordnungen, Mut und Tapferkeit, Bewaffnung der Männer?

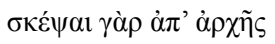

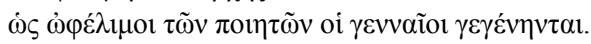

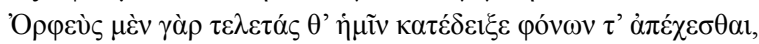

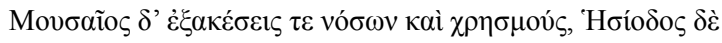

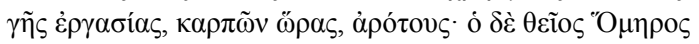

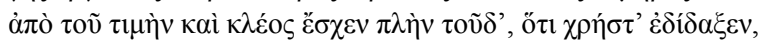

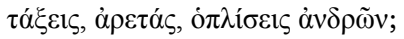

Und wenig später (Verse 1054-1056) resümiert er: „Für die kleinen Kinder ist es der Lehrer, der ihnen zeigt, wo es lang geht; für die Erwachsenen sind es die

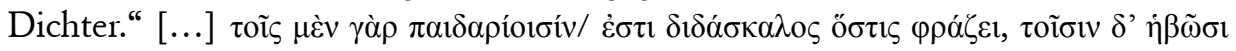

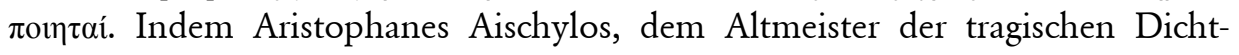
kunst, diese Funktionsbestimmung von Dichtung in den Mund legt, unterstreicht er einerseits die autoritative Geltung der Aussage, andererseits macht er jedoch deutlich, dass diese Meinung der Vergangenheit angehört und dass die ,modernen' Dichter wie Euripides eine ganz andere Auffassung haben.

Das Problem der Ununterscheidbarkeit von fiktional und nicht-fiktional durchzieht die homerische Odyssee, die in unmittelbarer zeitlicher Nähe zu Hesiods Lehrgedichten entstanden ist. ${ }^{4}$ Die zahlreichen Geschichten, die Odysseus erzählt, sind eine Mischung aus Wahrem und Fiktivem und erwecken somit den

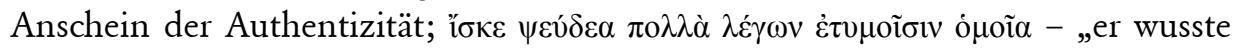
viel Erlogenes zu erzählen, das dem Wahren ähnlich war" - mit diesen, Hesiods Versen ähnlichen Worten kommentiert der epische Erzähler den Bericht, den Odysseus nach seiner Heimkehr nach Ithaka - noch unerkannt- seiner Frau Penelope gibt $(19,203)$. Der Dichter legt Odysseus eine Reihe von ,Trugreden' in den Mund, von autobiographischen Erzählungen, die der Protagonist nach seiner Heimkehr in mehreren Variationen zum Besten gibt, um seine wahre Identität zu verschleiern. Diese Geschichten sind im zeitgenössischen Kontext des frü-

4 Das zeitliche Verhältnis von Hesiods Lehrgedichten zu den homerischen Epen wird momentan neu durchdacht. Entgegen der früheren communis opinio wird heute häufig die Priorität von Hesiod angenommen; vgl. Reichel (2011: 28 f.). 
hen 7. Jahrhunderts v. Chr. durchaus ,plausibel', da sie den Erfahrungen der Griechen zur Zeit der aufkommenden Kolonisation und Handelsbeziehungen entsprechen; gleichzeitig enthalten sie - wie die autobiographischen Erzählungen in der ersten Hälfte des Epos - Selbstdeutungen des Odysseus. Er stellt sich in diesen Reden als reich, als Königssohn, als listig, mutig, unruhig und unstet und als wissbegierig dar, als eine Person, die wegen des Unverstandes der Gefährten in Ägypten viel Leid ertragen, Verschleppung und Versklavung, Irrfahrten und Schiffbruch erleiden musste. Dass Odysseus damit rechnen kann, mit seinen Trugreden ohne weiteres bei seinen Zuhörern Glauben zu finden, zeigt der Lebensbericht des Eumaios (15, 389-484), der ganz und gar wirklich Erlebtem entspricht und viele Berührungspunkte zu Odysseus' Truggeschichten aufweist: phoinikische Gauner, edle Abstammung, Verschleppung und Abenteuer zur See.

Die Trugreden des Odysseus verweisen insbesondere in den Reaktionen der Rezipienten auf das Hauptproblem, mit dem autobiographische Erzähler immer konfrontiert sind: die Glaubwürdigkeit des Erzählten. ${ }^{5}$ Wie kann der Rezipient sicher sein, dass das, was er hört oder liest, tatsächlich der Realität entspricht? Ausführliche Reflexionen darüber finden sich zu Beginn des 10. Buches (1-4) in Augustinus' Confessiones, der die Garantie für die Authentizität des in den vorangegangenen Büchern Berichteten in der caritas christiana, der ,christlichen Nächstenliebe', sieht, ${ }^{6}$ also, um Philippe Lejeunes Begriff zu verwenden, im ,autobiographischen Vertrag' (,pacte autobiographique“), dem stillschweigenden Abkommen zwischen Rezipienten und autobiographischem Text, ihn für ,wahr ${ }^{6} \mathrm{zu}$

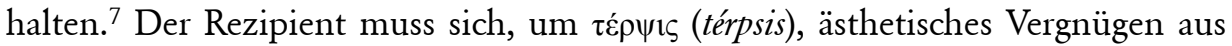
seiner Lektüre zu ziehen, auf den Pakt einlassen; nur ein außenstehender, gleichsam allwissender Leser kann zwischen wahr und fiktiv wirklich unterscheiden.

Diese, in der modernen Autobiographieforschung diskutierten Elemente finden sich durchaus mit metapoetischem Anspruch bereits in der Odyssee. Die allwissende Außenperspektive repräsentiert gleich in der ersten Lügengeschichte des Odysseus seine Schutzgöttin Athena, die Odysseus schmunzelnd darauf hinweist, dass seine Fabulierkunst und sein Listenreichtum sich zu wesentlichen Charaktereigenschaften herauskristallisiert hätten und sich als ein charakterliches Defizit erwiesen, das sein Wesen prägt (13, 29-297; vgl. 13, 254 f.). Am Hof des Königs Alkinoos auf der Insel der Phäaken, denen Odysseus als Sänger seines eigenen Lebens seine Irrfahrten nach der Eroberung Trojas in den so genannten Apologen (Bücher 9-12) vorträgt, wird der autobiographische Pakt zwischen Odysseus und seinen Zuhörern von beiden Seiten eingelöst - Odysseus kommt der Aufforderung des Alkinoos nach, wahrheitsgemäß der Reihe nach zu berichten $(12,572)$. Hingegen scheitert er teilweise im Falle des Schweinehirten Eumaios, bei dem Odysseus unerkannt nach seiner Heimkehr Unterschlupf findet, obwohl Odysseus gleich zu Beginn seiner

\footnotetext{
Vgl. dazu Holdenried (2000: 39-44) und Wagner-Egelhaaf (2000: 39-47).

Dazu ausführlich Zimmermann (2005: 243).

7 Lejeune (1975); deutsche Kurzfassung in Niggl (1989: 214-257).
} 
Geschichte deutliche Authentizitätssignale ausgesandt hatte (14, 192). Er scheitert, weil Eumaios der irrigen Meinung ist, selbst genaue Kenntnis von Odysseus' Schicksal zu haben, und deshalb Odysseus ironischerweise die Teile seiner Erzählungen nicht glaubt, die der Wahrheit am nächsten kommen (14, 363-366). Antinoos wiederum, einer der Freier, der um Penelopes Hand anhält, reagiert auf die Kurzfassung von Odysseus' Pseudobiographie (17, 415-444) ungehalten (17, 446452), da er sie wohl einer anderen Gattung zuweist, dem Ainos. Mit dieser im Epos und in der Lyrik der archaischen Zeit häufigen Erzählform soll im Rezipienten eine unmittelbare Reaktion ausgelöst werden: Er soll zu Handlungen bewegt werden, die er eigentlich nicht vorhat, oder dazu gebracht werden, dem Erzähler eines $A i$ nos ein Geschenk zu machen (vgl. 14, 508). ${ }^{8}$

Eine weitere Rezeptionshaltung biographischem und autobiographischem Erzählen gegenüber wird in Penelopes Reaktion deutlich. Wie nach dem Gesang des Sängers Phemios (1, 337-344), der von der glücklichen Heimkehr der Trojakämpfer berichtet, die ihrem sehnlichst erwarteten Mann bisher nicht zuteil wurde, ergeht es ihr beim Hören von Odysseus' Trugrede: Sie wird von Trauer und Trübsinn überwältigt und sogar von Schüttelfrost gepackt (19, 204-209). Diese psychosomatische Reaktion ähnelt der des Odysseus am Phäakenhof (8, 73-82; 8, 469-521): Er lauscht dort den beiden trojanischen Erzählungen des Sängers Demodokos. Die Ursache ist dieselbe: Wenn biographische oder autobiographische Erzählungen der Lebenserfahrung der Rezipienten zu nahe kommen, lösen sie nicht térpsis, ästhetisches Vergnügen, sondern - ganz der späteren Logos-Theorie des Sophisten Gorgias entsprechend (Diels/Kranz 82 B 2, 8 f.) - Erschütterung, Schauder und Tränen aus.

Die Faszination, die von Literatur ausgeht, und gleichzeitig das Misstrauen, das man ihr entgegenbringt, haben ihre Ursache neben der Ununterscheidbarkeit von wahr und falsch vor allem auch darin, dass Literatur alle möglichen Arten von emotionalen Reaktionen auslösen kann, wie dies vor allem die homerische Odyssee mehrfach vorführt. Dies gilt umso mehr, wenn sie, wie dies bis ins 4 . Jahrhundert hinein der Fall war, gehört, nicht gelesen und häufig als multimediales Ereignis dargeboten wurde, als Verbindung von Gesang, Musik, Gestik und Mimik und das in einer festlichen, aus der Welt des Alltags herausgehobenen Atmosphäre. Diese Macht des Logos, der Rede und Dichtung, treibt die Literaturtheorie der folgenden Jahrhunderte um.

Der Chorlyriker Pindar (ca. 518-440 v. Chr.) betont in seiner 7. und 8. Nemee, dass Odysseus' Ruhm zu Unrecht den des Aias bei weitem übertreffe, weil er in Homer einen „süßredenden“ Lobredner gefunden habe, der durch die Gewalt seiner Worte die Wahrheit verkehrt habe; ${ }^{9}$ denn „Meisterschaft täuscht, indem sie

8 Vgl. dazu M. J. Luzzatto (1996: 335).

9 Aias ist in der homerischen Ilias einer der wichtigsten griechischen Kämpfer vor Troja. Nach dem Tod des Achill hätten ihm aufgrund seiner Bedeutung und seiner verwandtschaftlichen Beziehung die Waffen des toten Helden zugesprochen werden müssen. Odysseus überredet 


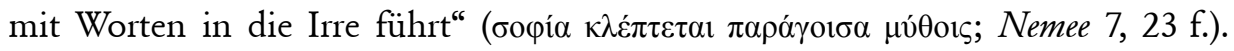
Literaturtheoretische Erwägungen sind, wie diese Bemerkungen Pindars, mit denen er eine Mythenkorrektur vorzunehmen versucht, deutlich vor Augen führt, immer als Auseinandersetzung mit Homer und Hesiod zu verstehen.

Die Omnipräsenz Homers in allen Bereichen des Lebens, insbesondere in der Erziehung ( $\pi \alpha_{1} \delta \varepsilon_{i} \alpha /$ paideía), in welcher Dichtung und vor allem die Aufführung von Dichtung in Tanz und Gesang eine herausragende Rolle spielte, forderte nicht nur die Kritik der Dichter, sondern schon früh die der dichtenden Philosophen heraus. Xenophanes von Kolophon (ca. 570-470 v. Chr.) prangert Homers schädlichen Einfluss an (Diels/Kranz 21 B 10): Er habe maßgeblich zu einer falschen Auffassung der Götter und des Göttlichen beigetragen. Alles hätten Homer und Hesiod den Göttern angehängt, was bei den Menschen als schändlich gelte (Diels/Kranz 21 B 11-12). Radikal lehnt er den Anthropomorphismus der homerischen Epen ab (Diels/Kranz 21 B 23-26). Für ihn sind die Götter, wie sie in den homerischen Epen erscheinen, Projektionen der menschlichen Vorstellung: Die Thraker stellten sich ihre Götter blauäugig und rothaarig, die Äthiopier schwarz und stumpfnasig vor (21 B 16 Diels/Kranz), und wenn Tiere malen könnten, würden sie ihre Götter als ihr eigenes Ebenbild entwerfen (Diels/Kranz 21 B 15). Wenn Heraklit von Ephesos (ca. 540-480 v. Chr.) dazu auffordert, Homer von den öffentlichen Wettkämpfen auszuschließen (Diels/Kranz 22 B 42), ist dies nur ein weiterer Beleg für die Dominanz Homers im kulturellen Leben Griechenlands: Bei einer Vielzahl von Agonen wurden seine Werke von den Rhapsoden rezitiert, wie dies Platons Ion vorführt, und prägten dadurch das Bewusstsein der Zuhörer, vor allem ihre Auffassung vom Göttlichen und den Göttern. Heraklit stellt vehement Homers Ruf als Weiser, als ernstzunehmender Philosoph, in Frage (Diels/Kranz 22 B 56).

Die philosophische Homerkritik wird zu Beginn des 5. Jahrhunderts durch eine Auseinandersetzung mit dem Dichter par excellence von Seiten der aufkommenden Geschichtsschreibung ergänzt. Hekataios von Milet (ca. 550-490 v. Chr.) setzt an den Anfang seines Geschichtswerks eine schonungslose Abrechnung mit Homer und seinen Rezipienten (FGrH 1, F 1a): ${ }^{10}$

Hekataios von Milet verkündet folgendes: Dies schreibe ich, wie es mir wahr zu sein scheint. Denn die Erzählungen der Griechen sind viele und lächerliche, wie sie mir erscheinen.

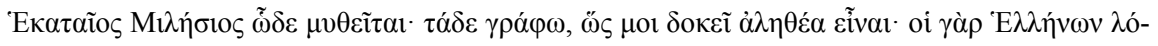

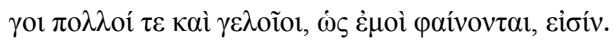

Eine Analyse der mythischen Erzählungen ergibt - so Hekataios -, dass das in ihnen Berichtete nicht der Realität entsprechen kann. Als Maßstab, um falsch und

die griechischen Heerführer, ihm die Waffen zuzusprechen. Enttäuscht will Aias Rache an den Anführern der Griechen nehmen, wird jedoch von Athena mit Wahnsinn geschlagen, so dass er statt der Griechen eine Schafherde niedermetzelt. Als er, aus dem Wahnsinn erwachend, seiner Tat gewahr wird, begeht er Selbstmord (vgl. Sophokles, Aias).

10 Vgl. dazu - mit ausführlichen Literaturhinweisen - Rengakos (2011: 331 f.). 
wahr zu unterscheiden, legt der erste Historiker seinen gesunden Menschenverstand an. Er überprüft die Überlieferung und schreibt nur das auf, was ihm wahr

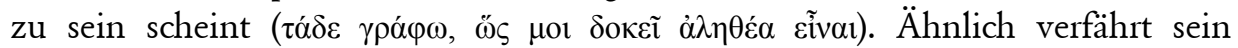
Nachfolger in der Geschichtsschreibung Herodot (ca. 490-420 v. Chr.). Häufig referiert er die verschiedenen lokalen Berichte, die ihm bei seinen Nachforschungen mitgeteilt wurden, überprüft sie nach Kriterien der Wahrscheinlichkeit und äußert dann seine eigene Meinung, was er für am wahrscheinlichsten hält. Thukydides (ca. 460-395 v. Chr.) schließlich betont in seinem Methodenkapitel in Absetzung von Herodot, dass er die Fakten nicht, wie es ihm richtig erscheine, sondern nur nach gründlicher Überprüfung aller ihm zur Verfügung stehenden Quellen berichte $(1,21)$. Den mythologischen Erzählungen vom trojanischen Krieg wohne - so der Historiker in seiner Frühgeschichte Griechenlands (1, 2-21) - ein historischer Kern inne, den man mit der von ihm praktizierten Methodik freilegen könne, indem man die Berichte - die Epen der archaischen Zeit - von ihrer dichterischen Ausschmückung befreie. Jede Art von Literatur - dies macht Thukydides deutlich - wird nach demselben Kriterium von wahr und falsch und letztlich nach dem Nutzen, den sie dem Rezipienten bringt, betrachtet und bewertet $(1,22,4)$.

Gerade diese Ununterscheidbarkeit von wahr und falsch machen sich die Sophisten, jene aus Platons Dialogen bekannten Redelehrer, in der zweiten Hälfte des 5. Jahrhunderts zunutze. Gorgias (ca. 480-380 v. Chr.) stellt die psychosomatischen Wirkungen des Logos, der Rede, und der Dichtung, die er als metrisch gebundene Rede definiert, in den Mittelpunkt seines Enkomions der Helena. ${ }^{11}$ Der Logos sei ein „großer Machthaber“, der, obwohl er mit einem äußerst kleinen und unscheinbaren Körper versehen sei, göttlichste Werke vollbringe. Denn er sei in der Lage, „Furcht zu beenden und Trauer zu beseitigen und Freude zu

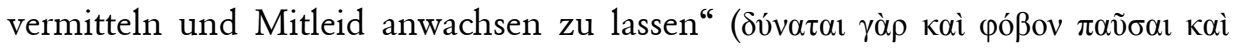

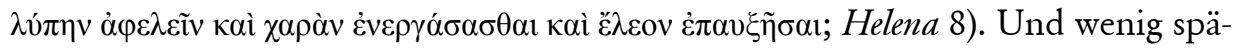
ter schreibt der Sophist (Helena 9):

Die Zuhörer überkommt, wenn sie diese [die Dichtung] hören, Schauder voller Furcht und Mitleid voller Tränen und leidliebendes Verlangen; ${ }^{12}$ bei Glücks- und Unglücksfällen fremder Angelegenheiten und Personen erleidet die Seele ein ganz eigenes Leid allein durch die Kraft der Worte.

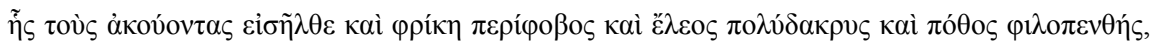

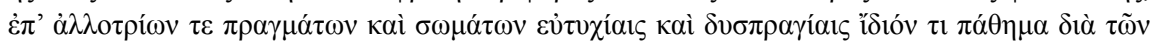
$\lambda \hat{\gamma} \gamma \omega v \ddot{\varepsilon} \pi \alpha \theta \varepsilon v \dot{\eta} \psi v \chi \eta \dot{~}$

Der Bereich, in dem der Logos diese Wirkungen entfaltet, sind nach Gorgias die

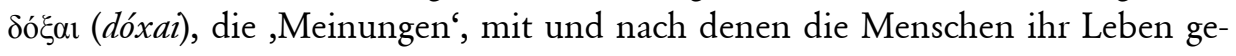

11 Am besten zugänglich bei Buchheim (1989: 3-16 Text und Übersetzung, 159-173 Kommentar).

12 Die auf den ersten Blick ein Oxymoron darstellende Verbindung ,leidliebendes Verlangen“ beschreibt das Verhalten eines Rezipienten, der, obwohl er weiß, dass er im Theater von allen möglichen Emotionen, auch von Leid, gepackt werden kann, sich trotzdem immer wieder diesen Affekten freiwillig und gern aussetzt. 
stalten, ohne mit letzter Gewissheit sicher zu sein, dass das, was sie gehört und gelernt haben und deshalb zu wissen glauben, tatsächlich so ist. Denn - so der Sophist - kein Mensch kann alles, was geschehen ist und was gerade geschieht und was geschehen wird - man beachte die Anklänge an Hesiod - wissen, so dass man sich für seine Meinungsbildung auf Informationen anderer verlassen müsse (Helena 11). Eine Person, die bei einem Sophisten wie Gorgias in die Lehre gegangen ist, kann - so der Werbezweck der kleinen Rede - seine Zuhörer am ehesten von etwas überzeugen und, zu was auch immer, überreden. ${ }^{13}$

Platons Literaturkritik setzt genau an diesem Punkt an. Die Gefahr, die von einer angenehm anzuhörenden, nach Mitteln der Kunst gestalteten Rede ausgeht, liegt auf der Hand: Die Vernunft wird ausgeschaltet; man fiebert mit den epischen und tragischen Helden mit, leidet mit ihnen, und es kann sogar, wie Platons Ion vorführt, zur Massenhysterie kommen, da diese Form des Literaturgenusses - von Dramen wie Epen - in der Regel immer Veranstaltungen vor einer großen Volksmenge waren. Die unterschiedlichen, von Gorgias beschriebenen Emotionen führen nach Platon zu einem Konflikt der Seelenteile und damit letztlich zur Unordnung im Staat (Politeia 606a-d). ${ }^{14}$ Die konsequente Ablehnung von Dichtung, von Homer und den Tragikern, die für Platon unter dem Gesichtspunkt des Stoffes (Mythos) und der Wirkung eine Einheit darstellen, gilt jedoch nur, wenn man sie als ,nützliche', als didaktisch-philosophische Texte liest, nicht wenn man sie als das betrachtet, was sie wirklich ist: als eine Art von ,Spiel' (Politeia 602b). Damit öffnet Platon - bei aller Kritik, die er an den herkömmlichen Dichtungsformen übt - eine neue Sicht auf Literatur. Literatur, die ,nützlich' sein kann, die den Rezipienten etwas lehren soll, muss nach anderen Maßstäben beurteilt werden als der Unterhaltung dienende Texte.

In direkter Auseinandersetzung mit Platon und der frühen philosophischen Dichtungskritik nimmt Aristoteles in der Poetik eine völlige Neubewertung der Dichtung vor. Indem er Mimesis (,Nachahmung') als einen der menschlichen Natur eigenen Trieb erklärt, öffnet er der Dichtung einen anthropologisch fundierten, autonomen Bereich. In der Modellhaftigkeit der dargestellten Situationen und Handlungen kann Dichtung dem Rezipienten Erkenntnis bringen und hat somit einen höheren ,Nutzen' als bloße Unterweisung. Eine ethischmoralische Dimension der Tragödie kommt durch die Dominanz zustande, die Aristoteles der Handlung (Mythos) und den in Handlungen involvierten Charakteren zuweist, die eine moralische Qualität besitzen und in ihren Erfolgen oder ihrem Scheitern vorgeführt werden. Deshalb gebührt Dichtung im Gegensatz zur

13 In dem Begriff $\pi \varepsilon \imath \theta \dot{~(p e i t h o ́) ~ m u ̈ s s e n ~ i n ~ d i e s e m ~ Z u s a m m e n h a n g ~ i m m e r ~ b e i d e ~ d e u t s c h e n ~}$ Übersetzungsmöglichkeiten, ,Überzeugung' und ,Überredung', mitgehört werden.

14 Vor allem wird Dichtung von Platon jedoch abgelehnt, da sie als Nachahmung der Abbilder der Ideen darstellenden Phänomene bereits zwei Stufen von den Ideen entfernt und deshalb minderwertig ist. 
Geschichtsschreibung das Epitheton ,philosophisch'. Die durch Dichtung, vor allem bei dramatischen Aufführungen ausgelösten Affekte deutet Aristoteles durch seine Katharsis-Konzeption positiv. Den die voraristotelische Literaturtheorie bestimmenden Gegensatz von wahr und falsch erweist die Poetik als eine falsche Herangehensweise an Dichtung, die nach anderen Kriterien (Wahrscheinlichkeit, Notwendigkeit, Handlungskonzeption) zu bewerten ist als ,Fachliteratur', in der Wahrheit und Richtigkeit der Aussagen das wichtigste Element sein sollten.

,Literaturtheoretische ${ }^{6}$ Äußerungen in der Zeit zwischen den homerischen Epen und der aristotelischen Poetik sind, wie der vorangehende Überblick zeigt, durch mehrere Komponenten bestimmt. In erster Linie gibt die Auseinandersetzung mit Homer die Themen vor, die die Diskussion bestimmen. Homer als der Dichter der Griechen (ó $\pi$ om $\tau$ ń $\varsigma)^{15}$ dominierte weit mehr den literarischen Diskurs, als dies andere Autoren taten. Der Grund hierfür ist darin zu sehen, dass man nur Homer in archaischer Zeit als, panhellenisch' bezeichnen kann, also als einen Dichter, der in der gesamten griechischsprachigen Welt rezipiert wurde. Andere Autoren wie die Lyriker und Chorlyriker hatten dagegen eher lokale Bedeutung (z. B. Sappho und Alkaios auf Lesbos, Archilochos auf Paros) und schrieben ihre Werke in der Regel als Auftragsdichtungen für ganz bestimmte Anlässe, vor allem Götterfeste. So wurde Homer schon früh zu dem wichtigsten Bezugstext, zum Lehrer aller Griechen, der ihr Weltbild und ihren Wertekanon prägte und der ihnen zusammen mit Hesiod, wie der Historiker Herodot schreibt $(2,53)$, die Götter und die Religion gegeben habe.

Die philosophische Kritik an Homer, die Xenophanes und Heraklit vorbrachten, richtet sich gegen diese Allgegenwart der Epen im Leben der Griechen, indem sie den Wahrheitsanspruch ins Spiel bringt. Was Homer an Unglaublichem berichtet, kann nicht der Wahrheit entsprechen; deshalb müsse man ihn - so Heraklit mit Nachdruck - aus dem öffentlichen Raum verbannen, zumal die gesprochene Rede, vor allem wenn sie künstlerisch, nach den Regeln der Rhetorik gestaltet ist, eine unheimliche Wirkung auf die Rezipienten auszuüben vermag: ${ }^{16}$

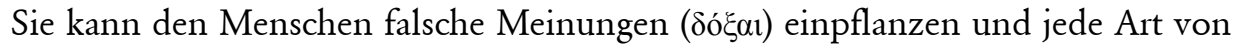
Affekt auslösen, wie dies die homerische Odyssee in den Sängerszenen mehrfach vorführt und wie es der Sophist Gorgias in seiner Helena theoretisch analysiert. Die Zuhörer würden, getäuscht durch die Macht der Rede, für wahr und real existierend halten, was ,erlogen' sei. Somit könne Dichtung nichts Nützliches sein, sondern höchstens eine Art von nutzlosem Zeitvertreib, geschweige denn irgendeine positive, didaktische Wirkung auf die Rezipienten ausüben - so Platons Resümee seiner Literaturkritik.

15 Unter Homers Namen waren eine Vielzahl von Epen im Umlauf, neben Ilias und Odyssee der sog. Epische Kyklos - epische Erzählungen, die sich um die beiden großen Epen rankten.

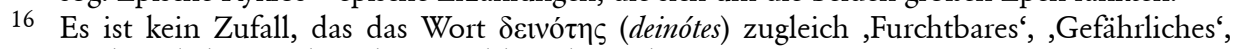
,Unheimliches' und ,Redegewandtheit' bezeichnet. 
Diese Stellungnahmen belegen eindrucksvoll, dass den Griechen bis ins 4. Jahrhundert v. Chr. hinein eine Unterscheidung von fiktionalen und faktualen Texten fremd war. ${ }^{17}$ Literatur hatte per se einen Wahrheitsanspruch, den es zu überprüfen galt. Die platonischen Dialoge führen immer wieder vor, wie diese autoritative Geltung Homers und Hesiods in Frage gestellt wird:18 Kann ein Feldherr mit der Ilias in der Hand tatsächlich eine Schlacht gewinnen? Weiß man, wenn man Hesiods Werke gehört hat, tatsächlich, was fromm ist oder wie man Ackerbau betreibt? Und vor allem: Wurde irgendein Mensch jemals durch Hesiods und Homers Epen moralisch besser?

Erst die aristotelische Poetik entzieht Literatur dieser in eine Sackgasse geratenen Diskussion, indem sie der Fiktionalität einen autonomen Raum öffnet und der schönen' Literatur einen höheren Wert zuschreibt, als ihn reine Sachtexte haben

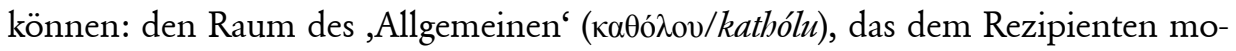
dellhaft Situationen des menschlichen Lebens, vor allem des menschlichen $\mathrm{Zu}$ sammenlebens, vorführt und deshalb durchaus eine moralisch-didaktische Wir-

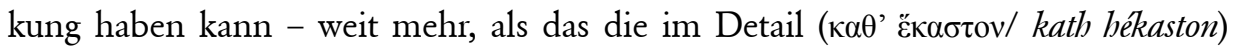
verhaftete Fachliteratur je leisten kann.

\section{Literatur}

\section{Ausgaben}

Aristophanes (2007) Aristophanis fabulae. Tomus II. Recognovit brevique adnotatione critica instruxit Nigel G. Wilson. Oxford: Oxford Univ. Press.

Aristoteles (1965) Aristotelis de arte poetica liber. Recognovit brevique adnotatione critica instruxit Rudolfus Kassel. Oxford: Clarendon Press.

Gorgias (1954) Die Fragmente der Vorsokratiker. Bd. I. Hgg. Hermann Diels und Walther Kranz. 7. Aufl. Berlin: Weidmann.

Buchheim, Thomas (1989) Gorgias von Leontinoi. Reden, Fragmente und Testimonien. Hamburg: Meiner.

Hekataios (1954) Die Fragmente der griechischen Historiker [1923]. Bd. I (FGrH). Hg. Felix Jacoby. Leiden: Brill.

Herodot (1908) Herodoti Historiae. Recognovit brevique adnotatione critica instruxit Carolus Hude. 2 Bde. Oxford: Clarendon Press.

Hesiod (1970) Hesiodi Theogonia, Opera et Dies, Scutum edidit Friedrich Solmsen. Oxford: Clarendon Press.

Homer (1908/13) Odyssee: Homeri opera recognovit brevique adnotatione critica instruxit Thomas W. Allen. Bd. III/IV. Oxford: Clarendon Press.

17 Vgl. dazu vor allem Rösler (1980).

18 Dies geschieht vor allem im Dialog Ion, in dem Sokrates den berühmten Epensänger Ion in die Enge treibt. 
Pindar (1971) Pindarus, Pars I: Epinicia post Brunomen Snell edidit Henricus Maehler. Leipzig: B.G. Teubner Verlagsgesellschaft.

Platon (1902) Politeia (Staat): Platonis opera recognovit adnotatione critica instruxit Ioannes Burnet, Tomus IV tetralogias VIII continens. Oxford: Clarendon Press.

Thukydides (1900/01) Thucydidis Historiae. Iterum recognovit brevique adnotatione critica instruxit Henricus Stuart Jones. 2 Bde. Oxford: Clarendon Press.

\section{Sekundärliteratur}

Holdenried, Michaela (2000) Autobiographie. Stuttgart: Reclam.

Lejeune, Philippe (1975) Le pacte autobiographique. Paris: Seuil.

Luzzatto, Maria Jagoda (1996) „Ainos [2, archaische Erzählform]”. Der Neue Pauly. Enzyklopädie der Antike. Bd. 1. Hgg. Hubert Cancik und Helmuth Schneider. Stuttgart/Weimar: J.B. Metzler. 335-36.

Niggl, Günter (1989) Hg. Die Autobiographie. Darmstadt: Wissenschaftliche Buchgemeinschaft.

Reichel, Michael (2011) „Das griechische Epos“. Handbuch der griechischen Literatur der Antike. Erster Band: Die Literatur der archaischen und klassischen Zeit. Hg. Bernhard Zimmermann. München: C. H. Beck. 7-78.

Rengakos, Antonios (2011) „Historiographie“. Handbuch der griechischen Literatur der Antike. Erster Band: Die Literatur der archaischen und klassischen Zeit. Hg. Bernhard Zimmermann. München: C. H. Beck. 326-417.

Rösler, Wolfgang (1980) „Die Entdeckung der Fiktionalität in der Antike“. Poetika 12: 283-319.

Wagner-Egelhaaf, Martina (2000) Autobiographie. Stuttgart/Weimar: Metzler.

Zimmermann, Bernhard (2005) „Exil und Autobiographie“. Antike und Abendland 48: 187-95. 
